\title{
An Approach for Optimizing the Shell Thickness of Core- Shell Quantum Dots using Photoinduced Charge Transfer
}

R. Vinayakan, ${ }^{\mathbf{a}}$ Shanmugha Priya, ${ }^{\mathbf{b}}$ Pratheesh V. Nair, ${ }^{\mathbf{a}}$ P. Ramamurthy ${ }^{\mathbf{b}}$ and K. George Thomas ${ }^{*}, \mathbf{a}$

${ }^{a}$ Photosciences and Photonics, National Institute for Interdisciplinary Science and Technology (CSIR), Trivandrum 695019, India

${ }^{b}$ National Centre for Ultrafast Processes, University of Madras, Taramani Campus, Chennai 600 113, India

Table of Content

Page No.

1. Instrumental methods and spectroscopic investigations

2. Synthesis of N-methylphenothiazine

3. Synthesis of TOPO capped CdSe QDs

4. Synthesis of TOPO capped CdSe/ZnS core-shell QDs

5. HRTEM Images of bare CdSe and ZnS capped CdSe QDs

6. Theoretical Calculation of band edge redox potential of CdSe QDs as a function of size

7. Effect of temperature and size of CdSe QDs on the photoluminescence quenching efficiency

8. TCSPC Analysis

9. References

S7

\section{Instrumental methods and spectroscopic investigations}

The electronic absorption spectra were recorded on a Shimadzu Model UV-3101, UV-VIS-NIR scanning spectrophotometer, emission spectra were collected using SPEX-Fluorolog F112X spectrofluorimeter and photoluminescence lifetimes were measured using an IBH Picosecond single 
photon counting system(luminescence decay profile was deconvoluted using IBH data station software V2.1). For HRTEM studies, a drop of nanoparticle solution was placed on a carbon coated $\mathrm{Cu}$ grid and the solvent was allowed to evaporate. Specimens were imaged on a JEOL $300 \mathrm{kV}$ high resolution transmission electron microscope. Cyclic Voltammetry was performed using a BAS CV50W Cyclic Voltammeter and tetrabutylammoniumhexafluorophosphate was used as supporting electrolyte. Organic compounds were characterized using $300 \mathrm{MHz}$ Bruker advanced DPX NMR spectrometer, JEOL JMS 600H mass spectrometer, Mel-Temp II melting point apparatus, Shimadzu FTIR spectrometer (IRPresige-21) and Shimadzu GCMS-QP2010 Gas chromatograph-mass spectrometer.

All the spectroscopic studies were carried out at ambient conditions (unless specified) where the QD concentration is in micromolar range. Quenchers, PT and NMPT were added as solutions, and also we have accounted for the small dilution effect $(<5 \%)$ by performing control experiments. QDs, both bare and ZnS overcoated were capped with mainly TOPO along with a lower percentage of TOP. We anticipate that in case of both bare and $\mathrm{ZnS}$ over coated QDs the organic capping layer of TOPO is being partly exchanged with PT resulting in the formation of a QD-PT complex.

\section{Synthesis of N-methylphenothiazine ${ }^{3}$}

Phenothiazine $(2 \mathrm{~g}, 10 \mathrm{mM})$ dissolved in dry THF $(15 \mathrm{~mL})$ was stirred with $\mathrm{NaH}(0.36 \mathrm{~g}, 15$ $\mathrm{mM}$ ) under argon atmosphere under dark for $1 \mathrm{~h}$ to yield the corresponding sodium salt. Methyl iodide $(1.9 \mathrm{~g}, 20 \mathrm{mM})$ was added, and the solution was further stirred for $24 \mathrm{~h}$ at room temperature to give N-methylphenothiazine, which was extracted with diethyl ether. The ether layer was dried using with anhydrous $\mathrm{Na}_{2} \mathrm{SO}_{4}$ and the solvent was evaporated and the crude product was chromatographed over neutral alumina using the solvent hexane to yield $90 \mathrm{mg}(42 \%)$ of $\mathrm{N}$ methylphnothiazine: $\mathrm{mp} 100-101{ }^{\circ} \mathrm{C}$; IR $(\mathrm{KBr}) v_{\max } 3739,3608,3055,2887,2818,2353,1782$, $1573,1556,1454,1330,1265,1138,1039,864,758,532.35,503,455,435 \mathrm{~cm}^{-1} ;{ }^{1} \mathrm{H} \mathrm{NMR}\left(\mathrm{CDCl}_{3}\right.$, 
$300 \mathrm{MHz}) \delta 3.37(3 \mathrm{H}, \mathrm{s}), 6.79-7.16$ (aromatic $8 \mathrm{H}, \mathrm{m}),{ }^{13} \mathrm{C} \mathrm{NMR}\left(\mathrm{CDCl}_{3}, 75 \mathrm{MHz}\right) \delta 35.31,114.04$, 122.46, 123.51, 127.18, 127.37, 145.83, $\mathrm{FAB}(\mathrm{m} / \mathrm{z}):\left(\mathrm{C}_{13} \mathrm{H}_{11} \mathrm{NS}\right) 213.06$.

\section{Synthesis of TOPO capped CdSe QDs ${ }^{1}$}

A reaction mixture containing $\mathrm{CdO}(0.067 \mathrm{~g}, 0.52 \mathrm{mM})$, dodecylamine $(3.8 \mathrm{~g}, 20.72 \mathrm{mM})$, trioctylphosphine oxide $(2.7 \mathrm{~g}, 6.9 \mathrm{mM})$ and tetradecylphosphonic acid $(0.40 \mathrm{~g}, 1.44 \mathrm{mM})$ was heated to $100{ }^{\circ} \mathrm{C}$, in an inert atmosphere. Then the reaction mixture was further heated to $300{ }^{\circ} \mathrm{C}$ until $\mathrm{CdO}$ dissolves completely to produce an optically clear solution. Keeping the temperature at $300{ }^{\circ} \mathrm{C}$, an injection mixture containing TOPSe $(83 \mu \mathrm{L}, 0.083 \mathrm{mM})$, TOP $(5.2 \mathrm{~mL}, 2.5 \mathrm{mM})$ was introduced. After desired crystal growth, the reaction was arrested by reducing the reaction temperature to ambient conditions and the QDs thus obtained were purified by size selective precipitation using methanol followed by centrifugation. In the present study we have synthesized CdSe QDs having three different sizes (3.3, 4.2 and $4.9 \mathrm{~nm}$ ) by controlling the crystal growth. QDs were characterized by HRTEM (Figure S1) and absorption spectroscopy.

\section{Synthesis of TOPO capped $\mathrm{CdSe} / \mathrm{ZnS}$ core-shell $\mathrm{QDs}^{2}$}

A reaction mixture containing TOPO, HDA and TOPO capped CdSe nanoparticles (concentration calculated based on UV-VIS spectroscopic studies) was heated to $100{ }^{\circ} \mathrm{C}$ in an inert atmosphere. The mixture was further heated up to $150{ }^{\circ} \mathrm{C}$ and an injection mixture containing calculated amount of $(\mathrm{Et})_{2} \mathrm{Zn}$ and $(\mathrm{TMS})_{2} \mathrm{~S}$ in TOP was added drop wise, under gentle stirring, over a period of half an hour. The reaction mixture was further cooled to room temperature and the $\mathrm{ZnS}$ capped CdSe core-shell QDs obtained were purified by reprecipitation using methanol. The purified product suspended in toluene was characterized by HRTEM analysis and absorption spectroscopy. (Figure S1). The number of monolayers was estimated as reported by Bawendi and co-workers. 
5. HRTEM images of bare CdSe and $\mathrm{ZnS}$ capped CdSe QDs

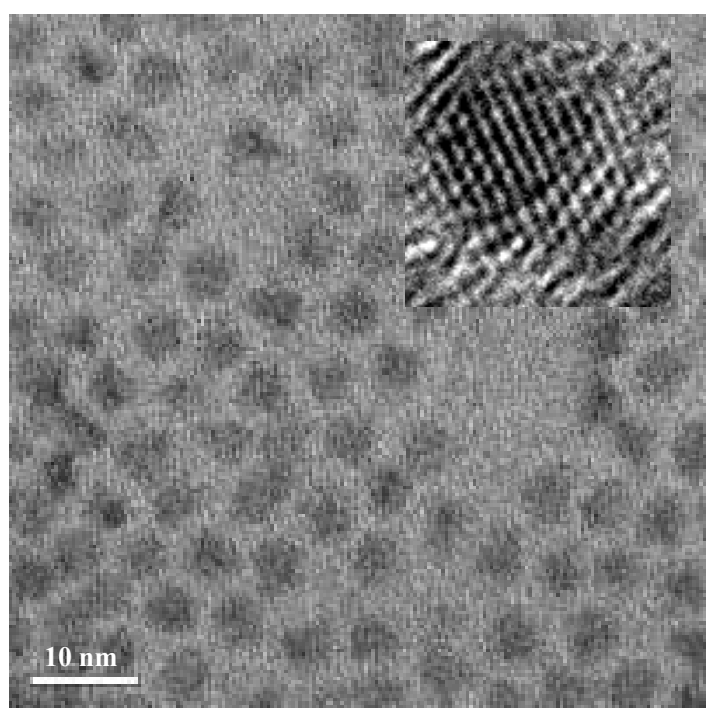

FigureSla: HRTEM images of bare CdSe QDs

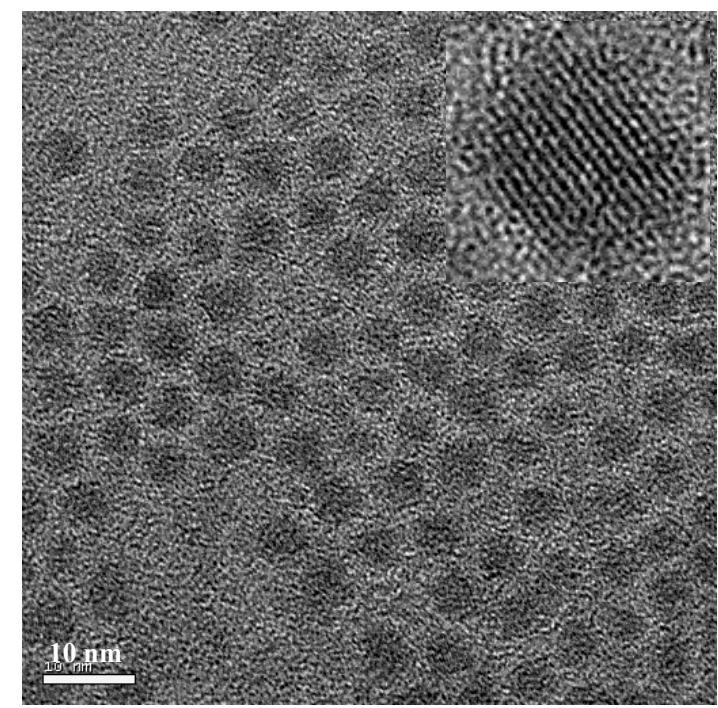

FigureSlb: HRTEM Image of CdSe QDs containing 2.6 monolayers of ZnS 


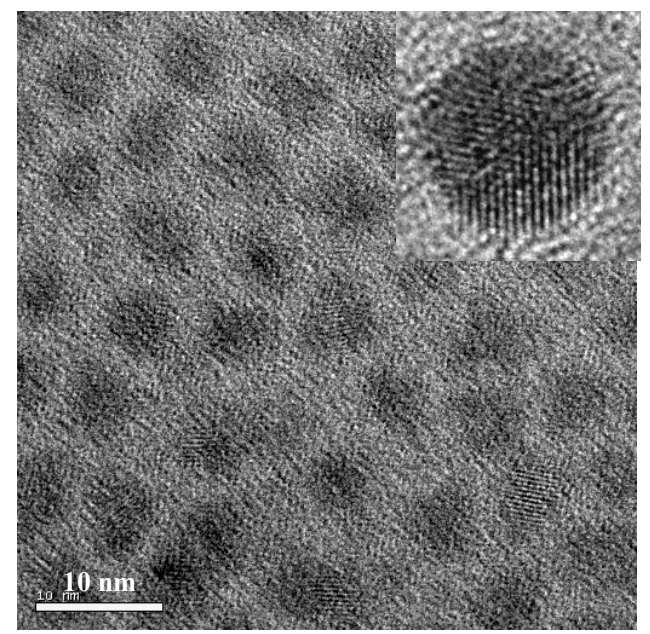

FigureS1c: HRTEM Image of CdSe QDs containing 3.9 monolayers of ZnS

\section{Theoretical calculation of band edge redox potential of CdSe QDs as a function of size ${ }^{4}$}

Absorbance spectra were used for estimating the shift in band gap energy due to quantum size effect and the respective valence and conduction band redox potentials. It is well known that the valence band and conduction band edge shifts to more positive and negative potentials respectively, due to quantum size effect. Following expression is used to calculate the relative change in the redox potentials of valence band $E_{\mathrm{VB}}$ and conduction band $\left(E_{\mathrm{CB}}\right)$ as a function of size $(\mathrm{R})$;

$$
\begin{aligned}
& E_{\mathrm{VB}}(\mathrm{R})=E_{\mathrm{VB}}^{\text {bulk }}+\Delta E_{\mathrm{VB}} \\
& E_{\mathrm{CB}}(\mathrm{R})=E_{\mathrm{CB}}^{\text {bulk }}-\Delta E_{\mathrm{CB}}
\end{aligned}
$$

For the calculations we have used well-known electrophysical constants for bulk CdSe; $E_{\mathrm{VB}}^{\text {bulk }}=+1.5 \mathrm{~V}$ against NHE, $E_{\mathrm{CB}}^{\text {bulk }}=-0.2 \mathrm{~V}$ against NHE. Results are summarized in Table 1.

Table 1: Calculation of band edge redox potential of CdSe QDs as a function of size

\begin{tabular}{cccccc}
\hline $\begin{array}{c}\text { Absorption } \\
\text { onset, } \mathbf{n m}\end{array}$ & $\mathbf{E}_{\mathbf{g}}(\mathbf{R}), \mathbf{e V}$ & Size, $\mathbf{n m}$ & $\boldsymbol{\Delta} \mathbf{E}_{\mathbf{g}}, \mathbf{e V}$ & $\begin{array}{c}\boldsymbol{E}_{\mathbf{C B}}, \mathbf{V} \text { vs. } \\
\mathbf{N H E}\end{array}$ & $\begin{array}{c}\boldsymbol{E}_{\mathbf{V B},}, \mathbf{V} \text { vs. } \\
\mathbf{N H E}\end{array}$ \\
\hline 524 & 2.4 & 3.3 & 0.66 & -0.70 & 1.66 \\
560 & 2.2 & 4.2 & 0.46 & -0.55 & 1.61 \\
590 & 2.1 & 4.9 & 0.36 & -0.47 & 1.58 \\
\hline
\end{tabular}


7. Effect of temperature and size of CdSe QDs on the photoluminescence quenching efficiency

Temperature dependent luminescence quenching studies for CdSe QDs, overcoated with 0.65 monolayers of $\mathrm{ZnS}$ is shown in Figure S2. A decrease in the static quenching constant was observed (' $\alpha$ ' at $298 \mathrm{~K}$ and $323 \mathrm{~K}$ are 174 and $96 \mathrm{M}^{-1}$ respectively) at elevated temperatures.

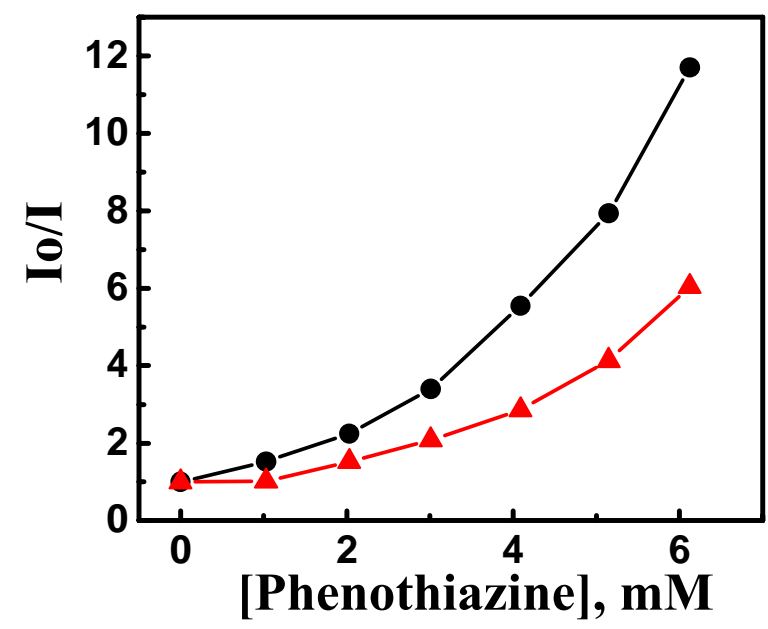

Figure S2: Changes in the relative photoluminescence intensity of CdSe QDs overcoated with $0.65 \mathrm{MLs}$ of $\mathrm{ZnS}$ on addition of PT at temperatures $(\bullet) 298 \mathrm{~K}$ and $(\boldsymbol{\Delta}) 323 \mathrm{~K}$.

We have also carried out photoluminescence quenching experiment with bare QDs of varying size $(3.2,4.2$ and $4.9 \mathrm{~nm})$ to probe the effect of core size on luminescence quenching and the results are shown in Figure S3. The interfacial charge separation was found to be insensitive to the core size of nanocrystals, an observation consistent with the relative redox potential level of valence band edge of $\mathrm{CdSe}$ and phenothiazine. (Note that the luminescence quenching by PT is more static in nature). 


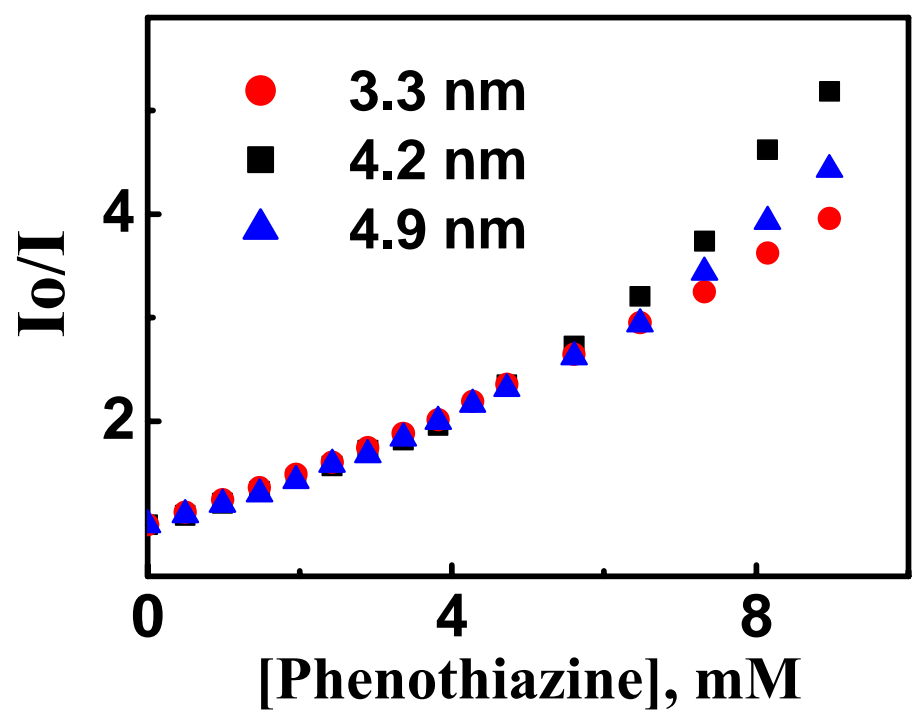

Figure S3: Photoluminescence quenching of CdSeQDs having varying core size in presence of $\mathbf{P T}$

\section{TCSPC Analysis ${ }^{5}$}

Average life times were determined using expression given below;

$$
\begin{aligned}
I(t) & =\sum \alpha_{\mathrm{i}} \exp \left(-\mathrm{t} / \tau_{\mathrm{i}}\right) \\
\tau_{\text {avg }} & =\frac{\alpha_{1} \tau_{1}^{2}+\alpha_{2} \tau_{2}^{2}+\alpha_{3} \tau_{3}^{2}}{\alpha_{1} \tau_{1}+\alpha_{2} \tau_{2}+\alpha_{3} \tau_{3}}
\end{aligned}
$$

Table2: TCSPC data for CdSe QDs in presence of phenothiazine

\begin{tabular}{cccccc}
\hline [PT], $\mathbf{m M}$ & $\boldsymbol{\tau}_{\mathbf{1}}, \mathbf{n s}$ & $\boldsymbol{\tau}_{\mathbf{2}}, \mathbf{n s}$ & $\boldsymbol{\tau}_{\mathbf{3}}, \mathbf{n s}$ & $\boldsymbol{\tau}_{\mathbf{a v g}}, \mathbf{n s}$ & $\chi^{\mathbf{2}}$ \\
0 & $4.0(16 \%)$ & $14(61 \%)$ & $46(23 \%)$ & 31 & 1.12 \\
2 & $3.9(17 \%)$ & $13(62 \%)$ & $43(21 \%)$ & 30 & 1.18 \\
4 & $3.7(18 \%)$ & $13(62 \%)$ & $43(20 \%)$ & 27 & 1.12 \\
6 & $3.9(18 \%)$ & $13(63 \%)$ & $44(19 \%)$ & 28 & 1.15 \\
8 & $3.8(16 \%)$ & $13(64 \%)$ & $50(20 \%)$ & 32 & 1.14 \\
\hline
\end{tabular}

\section{References}

1. Peng, X. A.; Peng, X. G. J. Am. Chem. Soc. 2001, 123, 183-184

2. (a) Dabbousi, B. O.; Rodriguez-Viejo, J.; Mikulec, F. V.; Heine, J. R.; Mattoussi, H.; Ober, R.; Jenson, K. F.; Bawendi, M. G. J. Phy. Chem. B 1997, 101, 9463-9475. (b) Kuno, M.; Lee, J. K.; Dabbousi, B. O.; Mikulec, F. V.; Bawendi, M. G. J. Chem. Phys. 1997, 106(23), 9869-9882. 
3. Sakaguchi, M.; Hu, M.; Kevan, L. J. Phy. Chem. 1990, 94, 870-874.

4. (a) Burda, C.; Green, T. C.; Link, S.; El-Sayed, M. A. J. Phys. Chem. B 1999, 103, 17831788. (b) Kucur, E.; Riegler, J.; Urban, G. A.; Nann, T. J. Chem. Phys. 2003, 119(4), 23332337.

5. Selmarten, D.; Jones, M.; Rumbles, G.; Yu, P.; Nedeljkovic, J.; Shaheen, S. J. Phys Chem. B 2005, 109, $15927-15932$. 